\title{
Agôn
}

Revue des arts de la scène

1 | 2008

Interstices, entractes et transitions

\section{A la recherche de la transition : Les intermèdes dans les traités de technique théâtrale italiens au XVII ${ }^{\mathrm{e}}$ siècle}

\section{Stéphane Miglierina}

\author{
(2) OpenEdition \\ Journals \\ Édition électronique \\ URL : http://journals.openedition.org/agon/708 \\ DOI : 10.4000/agon.708 \\ ISSN : 1961-8581 \\ Éditeur \\ Association Agôn
}

\section{Référence électronique}

Stéphane Miglierina, «A la recherche de la transition : Les intermèdes dans les traités de technique théâtrale italiens au XVIIe siècle », Agôn [En ligne], 1 | 2008, mis en ligne le 18 décembre 2008, consulté le 02 juin 2020. URL : http://journals.openedition.org/agon/708 ; DOl : https://doi.org/10.4000/agon. 708 


\section{A la recherche de la transition : Les intermèdes dans les traités de technique théâtrale italiens au XVII ${ }^{\mathrm{e}}$ siècle}

« La théorie n'est point difficile, mais plus facile encore est la pratique ». Cette phrase devenue célèbre par la citation récurrente qu'en a fait Louis Jouvet, sert d'épilogue au traité de conception des scènes de théâtre (au sens italien de décor puis de scénographie) de Nicola Sabbattini, Pratique pour fabriquer scènes et machines de théâtre (1638). ${ }^{1}$ Dans son exposé, Sabbattini présente, schémas à l'appui, et avec un souci d'exhaustivité, les différentes techniques architecturales et scénographiques disponibles à son époque ${ }^{2}$. S'il s'inscrit dans une tradition d'écrits théoriques à ce sujet, Sabbattini est sans aucun doute le plus concret et le plus pragmatique des auteurs de traités (trattatisti) de son époque : il apparait comme le fidèle observateur de la machine théâtrale en mouvement. Alors que le premier volume de son traité expose les techniques de construction des théâtres, le second montre au lecteur ces mêmes théâtres en mouvement. "Livre second - où il est traité d'intermèdes et de machines ». Le mot est lâché : l'intermède théâtral et sa lourde machinerie font l'objet de 56 chapitres détaillés (contre 41 dans le premier livre, traitant de la construction). C'est de cette présence insistante et récurrente de l'intermède dans les traités théoriques que naît notre réflexion sur la transition dans le théâtre du XVII ${ }^{\mathrm{e}}$ siècle. Sabbattini dès le titre du premier chapitre du second livre : «De la disparition et du changement des scènes $»^{3}$ fait de l'intermède le lieu même de la transition, du mouvement prompt et silencieux de changement du décor. Mais qu'en est-il de la pratique ? L'intermède peut-il véritablement être considéré comme le porteur de la fugacité lorsque l'on constate que dès la deuxième moitié du $\mathrm{XVII}^{\mathrm{e}}$ siècle et pour tout le XVIII ${ }^{\mathrm{e}}$ siècle il prend une ampleur considérable devenant un genre à part entière ?

\section{Débats sur l'intermède et polymorphie du genre}

Il importe de revenir avant tout à une définition de l'intermède. $\mathrm{Si}$, comme en témoignent les livrets du XVII ${ }^{\mathrm{e}}$ siècle ${ }^{4}$, la langue italienne compte plusieurs termes pour le désigner (intermedio, intermezzo, intromessa...) et si la réalité de l'intermède change au cours des décennies, il reste toujours cette composition plus ou moins brève située entre deux actes, voire également à la fin d'une pièce théâtrale, ayant pour but de varier le plaisir (diletto) du public. Défini par sa fonction plus que par sa forme, l'intermède, dans les livrets et les traités

\footnotetext{
${ }^{1}$ Nicola Sabbattini, Pratique pour fabriquer scènes et machines de théâtre, Neuchâtel, Ides \& Calendes, 1942.

${ }^{2}$ Même si en 1636 il entérine par son traité des techniques parfois déjà dépassées.

${ }^{3}$ Ibid., p.71.

${ }^{4}$ Cette étude est le fruit d'un croisement entre les traités de dramaturgie de la fin du XVI ${ }^{\mathrm{e}}$ et surtout du XVII ${ }^{\mathrm{e}}$ siècle et un ensemble de livrets théâtraux (comédies ou mélodrames) contenus dans le fonds Raccolta Drammatica Corniani Algarotti de la bibliothèque Braidense de Milan. A l'exception du traité de Sabbattini dont la traduction est celle de Louis Jouvet, Maria et Renée Canavaggia, toutes les traductions sont les nôtres.
} 
théoriques, est un genre polymorphique en évolution constante. Que 1'on cherche son origine dans les interventions du chœur antique ou dans les entremets, ces brefs spectacles de cour médiévaux représentées entre les plats d'un festin royal, l'intermède peut être chanté, mimé, dansé, joué ; il peut être comique, allégorique, historique voire religieux. Bref, ce lieu du changement est lui aussi en perpétuelle mutation et en perpétuelle croissance.

On l'a dit, l'origine de l'intermède est discutée, qu'elle soit antique ou médiévale. Les travaux d'Irène Mamczarz ${ }^{5}$ exposent clairement les différentes hypothèses à ce sujet pour dresser une typologie des intermèdes de la Renaissance au XVIII ${ }^{\mathrm{e}}$ siècle. Elle signale que dès la Renaissance la distinction est faite entre intermède apparente et non apparente, ce dernier étant un intermède uniquement musical alors qu'il est joué, dansé ou chanté lorsqu'il est dit apparente. Cette catégorie est toujours d'actualité dans la deuxième moitié du XVI siècle lorsque Leone De'Sommi écrit ses Quattro dialoghi in materia di rappresentazioni sceniche, traité de dramaturgie théorique où il expose sa définition de l'intermède :

Je dis que les intermèdes, en musique du moins, sont nécessaires aux comédies, tant en ce qu'il donnent une certaine fraîcheur à l'esprit des spectateurs, qu'en ce que le poète se sert de cet intervalle pour donner de l'ampleur à sa fable, puisque chacun de ces intermèdes, bien que bref, peut servir pour une durée de quatre, six ou huit heures afin que - bien que la comédie, si longue soit-elle, ne doive pas durer plus de quatre heures - souvent on la représente dans l'espace d'une journée entière, et parfois même de la moitié d'une autre ; et qu'il n'apparaisse pas de personnages en scène rend cet effet des plus efficaces.. ${ }^{6}$

De'Sommi défend ici l'intermède non visible, c'est-à-dire simplement ce repos de l'esprit qu'offre la musique sans comédiens mais il donne avant tout une définition de l'intermède qui est cet ajout à la pièce originale, permettant à la fois et de façon tout à fait paradoxale, d'allonger la représentation pour lui donner la proportion nécessaire et de la rendre supportable. Même s'il représente un ajout considérable au spectacle, l'intermède introduit un tel changement, un tel divertissement, une telle respiration qu'il est en mesure de soulager l'esprit du spectateur. Dès lors il découle que l'intermède, qu'il soit par ailleurs apparent ou non, bref ou long, est le porteur de la légèreté, du détournement de l'attention pour mieux retrouver la concentration du public lors de la pièce qu'il agrémente et enrichit. C'est pourquoi, parmi les différents types d'intermèdes, la veine qui prévaut jusqu'au XVIII ${ }^{\mathrm{e}}$ siècle, comme le souligne Mamczarc, est celle de l'intermède comique. En réalité, à partir de cette nécessité de «fraîcheur» de l'âme, un double mouvement historique s'opère : alors que les intermèdes sérieux, d'abord isolés de la fable vont être progressivement, au cours du siècle, intégrés à la pièce représentée pour prendre part à la fête théâtrale, l'intermède comique quant à lui prend de l'ampleur jusqu'à devenir un genre indépendant, semant ainsi les graines de l'opera buffa.

Le point de vue de De'Sommi permet également de souligner les temps de la représentation théâtrale de la fin du $\mathrm{XVI}^{\mathrm{e}}$ et pour tout le $\mathrm{XVII}{ }^{\mathrm{e}}$ siècle. Pour une fête théâtrale (terme plus adapté que représentation qui n'évoque qu'un seul type de spectacle) durant plusieurs heures voire jours, il n'est pas étonnant de constater que même une pièce ressentie

\footnotetext{
${ }^{5}$ Irène Mamczarz, Les Intermèdes comiques italiens au XVIIIe siècle en France et en Italie, Paris, Centre National de la Recherche Scientifique, 1972.

${ }^{6}$ « Dico che gl'intermedii di musica almeno, sono necessarii alle comedie, sì per dar alquanto di refrigerio alle menti de gli spettatori, et sì anco perché il poeta (...) si serve di quello intervallo nel dare proporzione a la sua favola, poscia che ognuno di questi intermedii, benché breve, può servire per lo corso di quattro, sei et otto ore, a tale che, quantunque la comedia, per lunga che sia, non ha da durar mai più che quattro ore, spesso se la dà spazio di un giorno intiero, et anco alcuna volta di mezzo un altro ; et il non comparire personaggi in scena fa questo effetto con maggiore eficacia. » Leone De'Sommi, Quattro dialoghi in materia di rappresentazioni sceniche, Milan, Il Polifilo, 1968, p.56. Nous soulignons.
} 
comme 'courte' ou 'légère' tel que l'intermède puisse devenir une représentation en soi, durant elle-même suffisamment de temps pour justifier les lourds changements de décor que décrit Sabbattini.

L'intermède est donc un moment de la fête marqué par la rupture, qu'elle soit d'ordre temporel (par un changement de rythme aussi bien que de durée des scènes représentées) ou d'ordre thématique. C'est ainsi que le comédien/dramaturge Niccolò Barbieri dans son traité La Supplica de 1634 souligne qu'il est coutume, surtout en France, de mêler œuvres sérieuses et intermèdes comiques (« Mais tous les comédiens d'outre-alpes font des œuvres graves avec intermèdes comiques» ${ }^{7}$ ). Ce changement de registre a toujours pour but au XVII ${ }^{\mathrm{e}}$ siècle, dans une optique baroque, l'émerveillement du spectateur (meraviglia), sa surprise et son éblouissement face à des formes théâtrales inattendues et inédites (ou du moins considérées comme telles). Ce n'est qu'à la toute fin du XVII ${ }^{e}$ siècle et pendant le XVIII ${ }^{e}$ siècle, avec l'apparition de l'Arcadie puis des Lumières, que la recherche de l'émerveillement cède la place à une certaine forme de réalisme ou du moins à un intermède marqué cette fois par le témoignage de la vie quotidienne et de la «qualité des temps ». Mais jusqu'alors, c'est avant tout la prouesse, la performance dirions-nous aujourd'hui avec anachronisme, qui est la valeur principale à l'aune de laquelle on juge les intermèdes.

Sabbattini nous montre à quel point une telle prouesse technique est valorisée lors des intermèdes : ainsi pour ne citer que quelques exemples de ces nombreux chapitres il explique « comment faire surgir des monts ou autres de sous le plancher de la scène », "comment représenter la transformation de pierres ou rochers en hommes » ou encore « comment faire descendre un nuage qui du fond du ciel vienne en avançant toujours jusques au milieu de la scène avec des personnes dessus $»^{8}$.

Mais plus encore que la théorie c'est la pratique qui nous renseigne sur les us de tels intermèdes ${ }^{9}$ : La Bradamante de Pietro Paolo Bissari, drame en musique, est publié à Venise en 1650 (et jouée probablement la même année au théâtre Grimano). Pièce en trois actes à thématique médiévale, elle est entrecoupée de deux intermèdes décrits de façon détaillée par le librettiste. Ces intermèdes ne sont pas signalés comme tels dans la typographie du livret mais le contenu disdascalique ne fait aucun doute quant à leur statut. On peut lire, à la fin du premier acte :

Sortie [sur scène] de Babouins qui, mis en fuite par des Chasseurs, par leurs farces forment la première partie d'un ballet.

La deuxième sera formée par les Chasseurs qui apparaissent entretemps, tandis que les Babouins cherchent à imiter leurs gestes.

La troisième, ils se croisent pour ne pas être attrapés. Au terme de cette partie les Babouins sautent sur les arbres et se dispersent. ${ }^{10}$

L'intermède introduit un élément d'exotisme propre au goût du XVII siècle (faisant apparaître chasseurs et babouins dans les forêts d'Europe du Nord), et une dimension grotesque au spectacle : les masques des singes, leurs farces dans la première partie du ballet

\footnotetext{
7 « Ma tutti i comici oltramontani fanno opere gravi con intermedi ridicolosi », Nicolò Barbieri, La Supplica, discorso familiare a quelli che trattano de' comici, Milan, Il Polifilo, 1971, p.54.

${ }^{8}$ Sabbattini, Nicola, Op. cit., p.107, 109 et 145.

${ }^{9}$ Nous nous limiterons à quelques exemples pratiques parmi la multitude de livrets de la Raccolta Drammatica Corniani Algarotti, les intermèdes de La Bradamante et de La Delia étant bien moins connus que d'autres intermèdes tels ceux de La Pellegrina.

${ }^{10}$ « All'uscir de' Babuini, che fuggiti da Cacciatori co'gli scherzi fatti trà essi formano la prima parte d'un Ballo. La seconda sarà con i Cacciatori, che vi sopragiungono, nel voler i Babuini imitare i loro gesti. La terza in trecia per non esser da essi presti [sic], per temine della quale salgono i Babuini sù par gli arbori, e si disperdono. » Pietro Paolo Bissari, La Bradamante, Venise, Valuasense, 1650, p. 30.
} 
puis l'imitation probablement toute comique des chasseurs dans la partie suivante, détournent l'esprit des spectateurs des amours de Bradamante et Ruggiero au cœur de l'intrigue. A cette maestria chorégraphique et technique, culminant dans l'évasion entre les arbres des singes dansants, succède, dans le second intermède, une prouesse technique qui n'est pas sans rappeler les chapitres de Sabbattini cités plus haut :

\begin{abstract}
Alors qu'Alcina est emportée par le Dragon volant, on offusquera la scène de plusieurs nuages, d'où seront sortis des Fantômes, qui, armés de verges lançant des flammes font des gestes incantatoires, et forment ainsi un Ballet. Y assistent des Esprits, qui surgissent des Airs, torches à la main, puis, après plusieurs farces et pas de danse, ils s'enfuient entre les Fantômes sans toucher terre. ${ }^{11}$
\end{abstract}

L'animal volant emportant la comédienne, l'apparition de spectres en vol puis leur disparition, la descente d'un personnage par les nuages, sont autant de techniques décrites dans la Pratique pour fabriquer scènes et machines de théâtre. La prouesse technique est volontiers alliée à l'accumulation d'effets visuels très spectaculaires pour créer cette meraviglia baroque tant attendue du public. Il faut noter une nouvelle fois que la fonction de l'intermède relève toujours de l'introduction de la légèreté dans le drame : aux dragons, aux fantômes portant des verges enflammées succèdent les petits esprits aux pas de danses légers et comiques.

Après de telles émotions mythologico-exotiques il convient de s'intéresser aux intermèdes de la Delia o sia la Sera sposa del Sole, de Giulio Strozzi (publié à Venise en 1639). La Delia est une pièce mythologique dont l'auteur explicite la symbolique dans un avertissement précédant le texte (associant, par exemple, les fils du soleil frappés par la foudre aux mortels accablés par le péché). La fin du premier acte est marquée par l'apparition d'un ballet de femmes puis d'hommes («Ballet des Dames de Délia et des Pages d'Admeto $\|^{12}$ ). Comme dans la majorité de livrets de l'époque, où l'indication d'une pièce musicale se résume à «s'en suit un ballet » ou « ballet masqué»" ${ }^{13}$, l'intermède dansé n'est pas explicité outre mesure. Il n'en va pas de même pour le second intermède qui cette fois est bien plus détaillé, cité ici dans sa langue originale pour comprendre le procédé d'anagramme chorégraphié.

Ballo di otto Soldati della guardia di Admeto, che formano negli scudi à lettere d'oro, questo Anagramma, e variandosi sempre nell'intreccio il colore d'un Turchino, ed'un Rosso, fanno con le cadenza riverenza alle Gentildonne

\begin{tabular}{|l|l|l|l|l|l|l|l}
1 & 2 & 3 & 4 & 5 & 6 & 7 & 8 \\
LA & BEL & TA & DE & RI & VE & RI & AMO
\end{tabular}

Anagramma Primo.

$$
\begin{array}{|l|l|l|l|l|l|l|l}
4 & 3 & 2 & 1 & 8 & 7 & 6 & 5 \\
\text { D E } & \text { TA } & \text { BEL } & \text { LA } & \text { AMO } & \text { RI } & \text { VE } & \text { RI }
\end{array}
$$

Rei pensieri, nembi dell'Alma,

Venti fieri, mentre danziamo,

Non turbate la nostra calma

LA BELTADE noi RIVERIAMO

Sia stabile il voler, se vola il piè :

S'intrecci la mano, s'impegni la fè.

Secol rio, cangiati homai :

\footnotetext{
${ }^{11}$ « Nel esser portata Alcina da Drago volante s'haverà offuscato la Scena con varie nubi, dalle quali saranno uscite Fantasme, che con verge, che vibran fiamme segnano i loro incanti, e dano [sic] forma a d'un Ballo. Assistono spiritelli, che dall'Aria sopragiungono con Faci alla mano, e doppo varij scherzi, e passi fuggono trà le Fanstame senza toccar la terra. » Ibid., p.56.

${ }^{12}$ « Ballo di Dame di Delia, e di Paggi d'Admeto », Giulio Strozzi, La Delia o sia La Sera sposa del sole, poema dramatico, Venise, G.P.Pinelli, 1639, p.32.

${ }^{13}$ « Segue il ballo.», « Ballo in maschera. »
} 
Donne, stelle terrene à vostri rai

Sian d'amanti Guerrieri

Principio D'ETA' BELLA AMORI VERI. ${ }^{14}$

Les boucliers des soldats dansants forment ainsi pendant tout l'intermède des anagrammes poétiques coordonnées à leur chant. Le poète décide avec minutie des couleurs et de leur alternance dans les costumes des soldats présentant chaque syllabe (ainsi il précisera pour l'anagramme suivant qu'il doit être tout d'une même couleur : " Anagramme Deuxième à dix soldats tous d'une seule couleur $»^{15}$ ). Prouesse poétique, cette fois, alliant la prosodie à la chorégraphie et à la conception des costumes, cet intermède n'est pas sans créer une vive émotion teintée d'émerveillement et de divertissement joyeux au sein du public. L'exigence de refrigerio dell'anima de De' Sommi est ici pleinement satisfaite puisque le rythme de l'intermède ainsi que les changements visuels imposés par le librettiste créent un effet de surprise par trois fois renouvelé à l'occasion des trois vers anagrammés.

On le voit, de la grandiloquence des chimères volantes à la préciosité des anagrammes chorégraphiés (mais nous aurions pu très bien nous arrêter également sur les intermèdes madrigaux du Pantalone Imbertonao, où chaque chanteur est associé à un instrument de musique et à un lieu de la fable ${ }^{16}$ ), l'intermède peut prendre des formes très variées. Plus qu'un recensement des types d'intermèdes, dont Irène Mamczarz s'acquitte avec minutie, il s'agit ici de souligner la polymorphie d'un genre en mouvement perpétuel. Face à cette polymorphie et surtout à son hypertrophie progressive, de nouvelles questions apparaissent. Si l'intermède occupe un temps toujours plus important lors de la fête théâtrale, il est loin d'être cet entre-acte, cet intervalle de non-jeu entre deux scènes jouées, puisqu'il est une " scène » à lui tout seul. La recherche de la transition dans le théâtre du XVII siècle se décale donc de l'alternance acte/acte à celle acte/intermède/acte. En d'autres termes, dès lors que l'intermède ne peut plus être considéré comme un moment de passage fugace mais comme un événement théâtral à part entière, que se passe-t-il entre la fin d'un acte et l'intermède ?

\section{Entre acte et intermède : la transition humanisée de Sabbattini.}

Les intermèdes qui portent réellement les marques de la transition entre deux actes sont très rares au XVII ${ }^{\mathrm{e}}$ siècle. Ainsi l'on peut trouver parfois une indication scénique pouvant

\footnotetext{
${ }^{14}$ [Ballet de huit soldats de la garde d'Admeto, qui de leurs boucliers aux lettres d'argents forment cet anagramme, et les déplaçant continuellement en se croisant on fera s'alterner le Bleu et le Rouge qui en rythme font la révérence aux nobles dames.

LA BEAUTE NOUS VENERONS.

Premier Anagramme

DU BEL AGE LES VRAIES AMOURS.

Pensées coupables, nuages de l'Âme

Vents féroces, alors que nous dansons

Vous ne troublez pas notre calme

LA BEAUTE NOUS VENERONS.

Que la volonté soit forte, si le pied est léger:

Que les mains se lient, que la parole s'engage.

Siècle perverti, il est temps que tu changes :

Dames, étoiles sur terre, que dans vos yeux

Les amoureux guerriers

Trouvent le début DU BEL AGE DES VRAIES AMOURS.], Giulio Strozzi, Op. cit., p.57.

${ }^{15}$ «Anagramma Secondo da dieci soldati tutti d'un colore» Ibid., p.58.

${ }^{16}$ Giovanni Briccio, Il Pantalone imbertonao, comedia nuova, Trevise, [s.e.], 1673.
} 
laisser supposer que le ballet intercalaire sert à vider la scène («Ballet de Pages qui emportent les tables du banquet ${ }^{17}$, nous dit-on dans l'intermède du Vespasiano de Giulio Cesare Corradi), mais de telles indications font exception. C'est à nouveau Sabbattini qui permet de trouver les premiers indices de la transition. Ainsi, dans son premier chapitre concernant les intermèdes il explique :

\begin{abstract}
Ayant déjà traité au cours du livre premier de la façon de construire des scènes et tout leur appareil nous allons à présent, en ce livre second, traiter des intermèdes et pour ce qu'il semble qu'on ne saurait, de nos jours, rien représenter de bon en ce genre si on ne change tout ou partie des scènes il m'a paru nécessaire de parler un peu de la façon de faire pareilles opérations car disparitions et changements de scènes sont en vérité de ces choses qui n'entraînent point, d'habitude, mince plaisir et vont émerveillant les spectateurs, singulièrement lorsque accomplies avec prestesse et sans que nul s'en avise ce qui, bien qu'assez mal aisé, arrive à s'obtenir toutefois grâce à la mise en œuvre d'un des divers artifices ci-après : comme de placer, tout exprès, une personne de confiance au fond de la salle laquelle, attentive à l'instant qu'il faudra changer de scène, aura l'air de se prendre alors de querelle avec une autre personne de connivence, ou feindra (mais ce pourrait être cause de désordres considérables) l'écroulement ou bris d'une quelconque poutre des gradins, ou encore par un coup de trompette, tambour ou autre instrument, divertira de la scène la vue des assistants ; cependant, la disparition aura lieu sans que nul s'en avise pourvu, toutefois, que l'on garde de laisser éventer semblable stratagème, lequel ne doit être découvert à personne, mis à part ceux qu'on y emploie.

De ces artifices le meilleur me parait celui de la trompette, ou d'un autre instrument, car ceux de la querelle ou du bris prétendu d'un gradin comportent maints périls, comme de faire naître quelque grand tumulte qui ne s'apaise ensuite aisément, tandis qu'au contraire une fois ouï, sans plus, le coup de l'instrument, comme dit plus haut, celui-ci s'étant tu, les gens se retourneront aussitôt vers la scène, comme devant et, recouvrant leur calme, admireront avec émerveillement et plaisir l'appareil nouveau présenté à leurs yeux. ${ }^{18}$
\end{abstract}

Sabbattini, avec un pragmatisme pouvant aujourd'hui faire sourire, décrit un moment de transition agitée parfaitement orchestré par l'organisateur de la fête (qui est appelé dans la traduction de Jouvet « ordonnateur » à plusieurs reprises). Inquiet de détourner l'attention des spectateurs (de le divertir en somme) aussi bien que de maintenir le calme nécessaire au bon déroulement de la représentation, il privilégie la surprise musicale plutôt que le faux incident technique ou la prétendue rixe. Parmi les valeurs qu'il prône pour une bonne transition entre acte et intermède la vitesse est la première, elle est alliée au silence (puisque personne ne doit se rendre compte des changements de décor) et au secret de la technique, sans lequel la diversion ne pourrait avoir lieu. C'est par l'alliance de ces trois conditions que le plaisir naitra dans l'esprit du spectateur. Ces valeurs sont récurrentes tout au long de son traité. Il faut " exécute[r] la manœuvre, doucement et sans bruit »", où 'doucement' est à comprendre comme 'sans à-coups', 'avec légèreté' ; il faut faire en sorte que tout se passe « de façon plus sûre et avec moindre risque de désordre.»”

Dans ce premier chapitre du second livre sur les intermèdes, Sabbattini fait apparaître au détour d'une phrase l'élément fondamental au bon déroulement de la transition : « ceux qu'on y emploie ". Si son traité est si populaire auprès des techniciens de théâtre (y compris contemporains) ce n'est pas uniquement pour ses qualités mécaniques : il est sans aucun doute le premier à dresser le portrait (même allusif) et à donner une place importante aux hommes de main du théâtre. Architecte et peintre, certes, mais aussi et surtout manœuvres opérant en coulisses. La transition vers l'intermède est le moment où l'on entre-aperçoit l'ombre des techniciens, qu'il appelle simplement « hommes » ou «gens ». Sabbattini en fait un portrait

\footnotetext{
17 « Ballo de’ paggi che levano le mense » Giulio Cesare Corradi, Il Vespasiano drama per musica, Venise, Francesco Nicolini, 1680, p.31.

${ }^{18}$ Nicola Sabbattini, Op. cit., p. 71-72.

${ }^{19}$ Nicola Sabbattini, Op. cit., p. 82.

${ }^{20}$ Nicola Sabbattini, Op. cit., p. 81.
} 
idéal, les classant tacitement en deux catégories. Ainsi le «bon » technicien doit être avant tout consciencieux. Pour bien refermer les trappes dans la scène après l'entrée d'un danseur

il conviendra que les hommes qui auront le soin des étais remettent ceux-ci en place avec l'aide des autres hommes, comme fut fait pour l'ouverture, et ainsi mènera-t-on à bien ladite opération, pourvu aussi que l'on ne manque d'y employer des gens qui y soient propres et s'en fassent un point d'honneur afin que le tout ait lieu en bon ordre. ${ }^{21}$

En d'autres termes ils devront être «bonnes gens, mettant zèle et honneur à bien faire $\gg{ }^{22}$ A leur droiture morale devrait s'allier une force physique conséquente afin de pouvoir soulever ou tirer les éléments du décor : "on mettra de chaque côté deux hommes lesquels devront être vigoureux et râblus $» .^{23}$ Ils doivent être capables de la plus grande concentration alors même que la diversion du public s'opère : "Ils devront tirer, sans rien considérer d'autre, jusques aux signes qu'il aura fallu marquer. » ${ }^{24}$ Enfin, « il faut, pour ce, grand entendement et vigilance tant de part de qui ordonne comme encore de qui exécute. ${ }^{25}$ Sabbattini pose ainsi les bases d'un pacte de confiance entre l'organisateur du spectacle et ses techniciens, pacte de confiance qui est à la base du bon fonctionnement de la représentation. Sabbattini fait ce portrait idéal d'un technicien vertueux qui s'oppose par ailleurs à sa contrepartie inefficace, moins présente : « il est besoin en ces actions de bien prendre garde (...) aussi ne doivent-elles être exécutées par des personnes sottes et balourdes.» ${ }^{26}$

L'apport de Sabbattini à la compréhension de la pratique de la transition est donc fondamental en ce qu'il humanise l'entre-acte. Alors que l'intermède peuple la scène de chanteurs, de danseurs, de musiciens, l'entre-acte-intermède et sa description laissent apercevoir les hommes des coulisses, ce hors-scène idéal, où tout mécanisme est bien rodé, où chacun est à sa place, et accomplit sa tâche avec application. Une telle optique n'est pas sans rappeler les conceptions d'Ancien Régime que Sabbattini semble appliquer ici à la pratique du théâtre, microcosme social même dans son fonctionnement mécanique. Au Prince, au père, au gouverneur, tant de figures paternelles d'une société fondée sur le cercle clos d'une hiérarchie de type familiale, s'ajoute ici l'ordonnateur du spectacle, qui lui aussi gouverne ses hommes avec fermeté et justice, offrant à chacun le rôle qui lui est le plus adapté, et chacun de l'accepter et de l'honorer avec respect. C'est ainsi que la notion de devoir s'ajoute enfin aux autres valeurs morales prônées par Sabbattini : " ainsi aura-t-on fait tout ce qu'il fallait » répète-t-il à la fin de nombreux chapitres. Ce devoir lui apparaît comme une évidence face à un défi architectural à relever, et disparaît dès lors qu'est achevée l'explication mécanique adressée au lecteur aguerri. Ce lecteur lui-même entre dans le pacte de confiance que Sabbattini met en avant: il postule la présence face à lui d'un lecteur idéal, au fait des pratiques théâtrales et des habitués des schémas techniques. "Ici point ne se met de figure pour ce que c'est affaire tant ordinaire qu'il ne se trouve quasi personne, n'ayant, en son logis, les lumières qu'il faut.» ${ }^{27}$

Intermèdes, entracte et transitions sont ainsi liés dans la réflexion d'ordre dramaturgique du XVII ${ }^{\mathrm{e}}$ siècle. L'intermède, scène de passage entre deux actes, devenu genre

\footnotetext{
${ }^{21}$ Nicola Sabbattini, Op. cit., p. 98-99.

${ }^{22}$ Nicola Sabbattini, Op. cit., p. 105.

${ }^{23}$ Nicola Sabbattini, Op. cit., p. 101.

${ }^{24}$ Nicola Sabbattini, Op. cit., p. 137.

${ }^{25}$ Nicola Sabbattini, Op. cit., p. 103-104.

${ }^{26}$ Nicola Sabbattini, Op. cit., p. 105-106.

${ }^{27}$ Nicola Sabbattini, Op. cit., p. 108.
} 
à part entière est une forme polymorphique dont la fonction est liée au changement de rythme, aussi bien que de ton ou de registre. Il doit apporter le divertissement nécessaire au public pour affronter l'acte suivant avec l'esprit «rafraîchi » et dès lors, il est l'occasion de prouesses techniques et artistiques menant à la surprise et l'émerveillement baroque. Cependant, alors qu'au fil du siècle, l'intermède, particulièrement dans sa forme comique, prend une ampleur toujours plus considérable, il perd de sa fonction de transition, et c'est dans l'alternance acte-intermède-acte qu'il faut chercher l'intervalle de l'entracte, celui-là même qui est nécessaire à l'installation des lourds décors et machineries de l'intermède. Dans ce moment du non-vu, de la distraction du public, apparaissent les silhouettes en coulisses de proto-techniciens valorisés par les traités théoriques. Si peu nombreux sont les textes des intermèdes qui font allusion à de tels hommes de main (on pense à l'Intermedio per una commedia de Carlo Maria Maggi en 1697 où la récitante fait allusions aux habilleuses qui ont trop serré les lacets de son corset), les traités de technique théâtrale et en particulier celui de Sabbattini laissent une place ponctuelle mais récurrente à la hiérarchie des hommes de théâtre. Là où il dessine les schémas de machineries complexes, Sabbattini esquisse également la miniature de la structure sociale qui dans laquelle il évolue.

\author{
Stéphane Miglierina \\ Doctorant à l'Université Paris VIII - Saint-Denis \\ stephane miglierina@hotmail.com
}

\title{
Bibliographie
}

Il Corago o vero, alcune osservazioni per metter bene in Scena le composizioni drammatiche, Fabbri Paolo et Angelo Pompilio (dir), Florence, Olschki, 1983.

Trattatisti e narratori del Seicento, Milano-Napoli, Ricciardi, 1960

Allacci, Leone, Drammaturgia, Roma, Mascardi, 1666.

Barbieri, Niccolò, La Supplica, discorso famigliare a quelli che trattano de'comici, Ferdinando Taviani (dir.), Milan, Il Polifilo, 1971.

Bartolommei, Girolammo, Didascalia cioe Dottrina comica, Florence, S.A.S. alla Condotta, 1661 .

Bissari, Pietro Paolo, La Bradamante, drama per musica, Venise, Valuasense, 1650.

Briccio, Giovanni Il Pantalone imbertonao, comedia nuova, Trevise, [s.e.], 1673.

Carini Motta, Fabrizio, Trattato sopra la stuttura de' theatri e scene che a'nostri giorni si costumano, E.A.Craig (dir.), Milan, Il Polifilo, 1972.

Corradi, Giulio Cesare, Il Vespasiano, drama per musica, Venise, Francesco Nicolini, 1680.

De'Sommi, Leone, Quattro dialoghi in materia di rappresentazioni sceniche, Ferrucio Marotti (dir.), Milan, Il Polifilo, 1968.

Maggi, Carlo Maria, Il teatro milanese, Turin, Einaudi, 1964.

Mamczarz, Irène, Les Intermèdes comiques italiens au XVIIIe siècle en France et en Italie, Paris, Centre National de la Recherche Scientifique, 1972.

Sabbatini, Niccolò, Pratique pour fabriquer scènes et machines de théâtre, Louis Jouvet (dir.), Neuchâtel, Ides et Calendes, 1942.

Strozzi, Giulio, La Delia o sia La Sera sposa del sole, Venise, G.P. Pinelli, 1639. 


\section{Références de l'article :}

Stéphane Miglierina, «A la recherche de la transition : Les intermèdes dans les traités de technique théâtrale italiens au XVII ${ }^{\circ}$ siècle», Agôn [En ligne], $\mathrm{N}^{\circ} 1:$ Interstices, entractes et transitions, Dossiers, Dramaturgies de l'interstice, mis à jour le : 05/12/2008, URL : http://agon.ens-lsh.fr/index.php?id=708. 